\title{
A Reconfigurable Cache Architecture for Energy Efficiency
}

\author{
Karthik T. Sundararajan \\ School of Informatics \\ The University of Edinburgh \\ United Kingdom \\ t.s.karthik@ed.ac.uk
}

\author{
Timothy M. Jones \\ Computer Laboratory \\ The University of Cambridge \\ United Kingdom \\ timothy.jones@cl.cam.ac.uk npt@staffmail.ed.ac.uk
}

\begin{abstract}
On-chip caches often consume a significant fraction of the total processor energy budget. Allowing adaptation to the running workload can significantly lower their energy consumption. In this paper, we present a novel Set and way Management cache Architecture for efficient Run-Time reconfiguration (Smart cache), a cache architecture that allows reconfiguration in both its size and associativity. Results show the energy-delay of the Smart cache is on average $18 \%$ better than state-of-the-art reconfiguration architectures.
\end{abstract}

\section{Categories and Subject Descriptors}

B.3.2 [Memory Structures]: Cache memories

\section{General Terms}

Management, Performance, Experimentation

\section{Keywords}

Configurable cache, Cache tuning, Smart cache

\section{INTRODUCTION}

The power dissipation of modern microprocessors is a primary design constraint across all processing domains, from embedded devices to high performance chips. Cache memories contain a large number of transistors and consume a large amount of energy. Customization of cache parameters may be static or dynamic; in a static approach the designer sets the cache parameters before synthesis, whereas in a dynamic scheme the cache parameters can be modified within a certain range to adapt to the running application.

This paper proposes a configurable cache architecture that allows reconfiguration of both the size and associativity, providing maximum flexibility to the application. We compare our approach, called the Smart cache, against stateof-the-art cache reconfiguration techniques and show that our scheme's energy-delay product is on average $14 \%$ better than these prior works.

\section{THE SMART CACHE ARCHITECTURE}

This section describes the Smart architecture that we use for the level-2 cache within the system. Figure 1 shows how

Copyright is held by the author/owner(s). CF'11, May 03-05, 2011, Ischia, Italy. ACM 978-1-4503-0698-0/11/05.

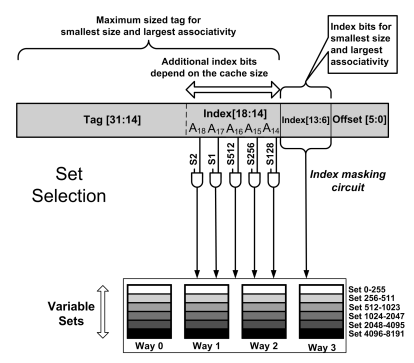

(a) Set Selection

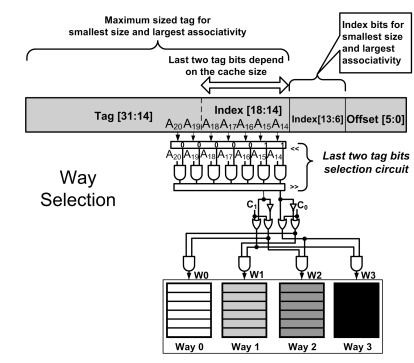

(b) Way Selection
Figure 1: Organization of the Smart cache architecture. Varying the cache size (through the set selection circuits) is performed in parallel with altering the associativity (through the way selection logic).

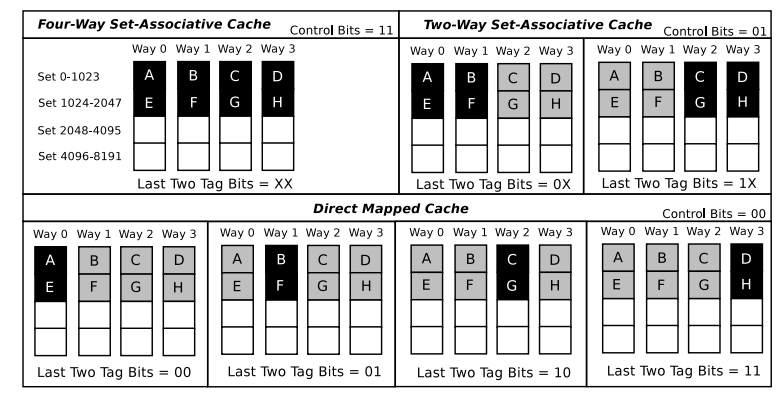

Figure 2: Behavior of the Smart cache. Black, grey and white regions show accessed, unaccessed and disabled sets respectively. Control bits determine the associativity and the last two tag bits determine the ways.

each address is mapped onto a $2 \mathrm{MB}$ level- 2 cache. There are two complementary circuits used in parallel that perform the mapping to route the address to the correct set and way.

We group the sets in each bank by augmenting the cache with size selection bits that determine the sets that are enabled. These are then $A N D$ ed with bits from the index to determine the sets to access. A $64 \mathrm{~KB}$ cache (sets 0-255) is always enabled even when all size selection bits are 0 .

In order to control the associativity of the cache, we augment it with a way selection circuit. This uses the last two tag bits to route accesses to the ways that are enabled. Since the cache size can vary, the size selection bits are required to correctly identify the last two tag bits. 


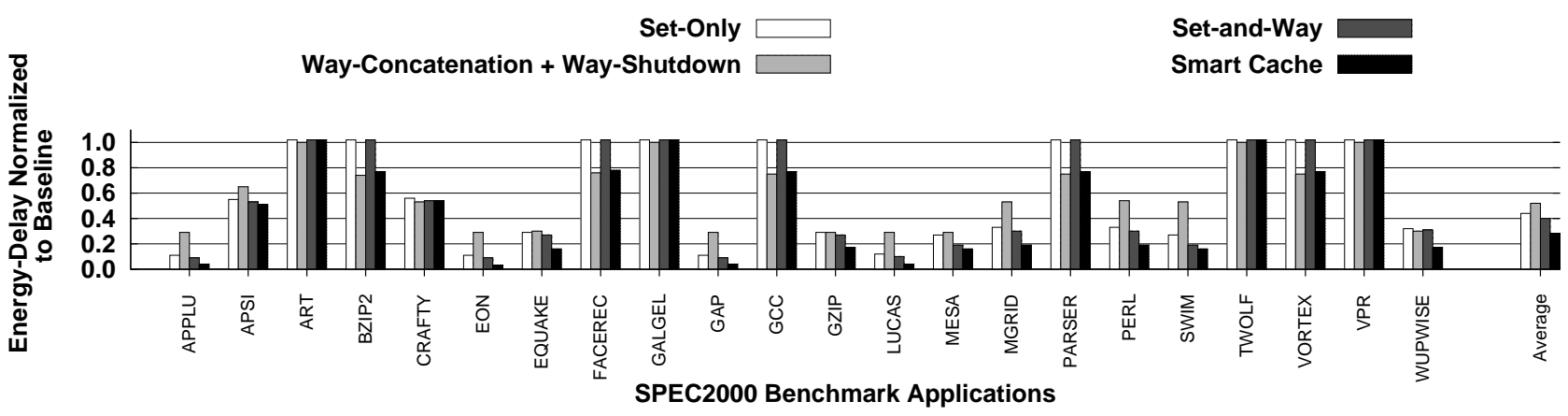

Figure 3: Energy-delay values for different cache architectures running on the same L2 baseline cache.

As the cache size and associativity varies, so does the number of bits needed for the tags. In our architecture, we store the maximum sized tag for each line (i.e., for the smallest cache and largest associativity). Figure 2 shows how the control and tag bits map to the ways that are enabled in our Smart cache.

\subsection{Overheads}

The way selection circuit does not appear in the cache's critical path because it can operate in parallel with the tag and data array address decoders [5]. The set selection circuit can be folded into the decoders to avoid any delay in calculating the index bits [1]. Therefore there is no increase in the cycle time for accessing the cache using our approach. However, after reconfiguring the cache to a smaller size, we turn off unused sets and ways, destroying their contents. Therefore we must flush any dirty lines back to the next level in the memory hierarchy. In all our simulations we add the flushing cost that includes both power and performance costs for copying back the dirty lines. We have calculated the area overheads of the cache as $0.5 \%$ over the baseline. This is due to the extra control circuitry required to perform set selection, way selection and reconfiguration.

\subsection{Relation to Prior Work}

There are several key differences between our Smart cache and state-of-the-art reconfiguration techniques. In our approach the associativity and size are varied in parallel by using the way control signals and the size control registers. The Smart cache organizes ways at set boundaries, which avoids flushing data back to memory when increasing the associativity but keeping the cache size fixed. This addresses the shortcomings in previous techniques [5], allowing dynamic reconfiguration of the cache. In addition to this, the Smart cache offers 3x more cache configurations than the set-only [3] and hybrid [4] schemes which combine way-concatenation with way-shutdown.

\section{RESULTS}

This section evaluates our Smart cache approach against prior cache architectures on static configurations of the level2 cache. We implemented these approaches in the HotLeakage simulator [6], updating the underlying power models to use a more recent version of Cacti (v5.3) [2] that has been modified to support our new circuitry for $70 \mathrm{~nm}$ process technology. We also altered the simulator to include the power and performance overheads of reconfiguring each cache. We modelled an Alpha out-of-order superscalar whose cache configurations are similar to an Intel Core 2 processor.

Figure 3 shows the energy-delay values achieved when running each benchmark on the best static configuration for that application for each cache architecture. The best static configuration is the one that has the lowest energy-delay and a maximum $2 \%$ performance loss, from all the possible configurations. Figure 3 shows on average level- 2 cache energy-delay achieved by our approach is 0.24 , which is $14 \%$ better than set-only cache [1], set-and-way cache [4] and $25 \%$ better than the way-concatenation combined with the wayshutdown cache [5]. This clearly demonstrates the benefits of using our Smart cache architecture for run-time cache reconfiguration.

\section{CONCLUSIONS}

This paper has presented a novel configurable cache architecture that can be reconfigured in both size and associativity to match the requirements of an application. We have demonstrated that our Smart cache architecture has an energy-delay product that is on average $18 \%$ better than state-of-the-art approaches. Future work will harness this architecture for dynamic cache adaptation to fit the cache to the requirements of each application phase as it runs.

\section{REFERENCES}

[1] M. Powell et al. Gated-vdd: a circuit technique to reduce leakage in deep-submicron cache memories. In ISLPED, 2000.

[2] S. Thoziyoor et al. Cacti 5.1. Technical Report HPL-2008-20. HP Laboratories Palo Alto, 2008.

[3] S.-H. Yang et al. Dynamically resizable instruction cache: An energy-efficient and high-performance deep-submicron instruction cache. Purdue University, 2000.

[4] S.-H. Yang et al. Exploiting choice in resizable cache design to optimize deep-submicron processor energy-delay. In $H P C A, 2002$.

[5] C. Zhang et al. A highly configurable cache architecture for embedded systems. ISCA, 2003.

[6] Y. Zhang et al. Hotleakage: A temperature-aware model of subthreshold and gate leakage for architects. Technical Report,CS-2003-05, 2003. 